\title{
Novel function of E26 transformation-specific domain-containing protein ELK3 in lymphatic endothelial cells
}

\author{
JI-IN PARK ${ }^{1}$, KWANG-SOO KIM $^{1}$, SUN-YOUNG KONG ${ }^{2-4}$ and KYUNG-SOON PARK ${ }^{1}$ \\ ${ }^{1}$ Department of Biomedical Science, College of Life Science, CHA University, Seoul 13488; ${ }^{2}$ Department of \\ System Cancer Science, Graduate School of Cancer Science and Policy, National Cancer Center; \\ ${ }^{3}$ Translational Epidemiology Branch; ${ }^{4}$ Department of Laboratory Medicine, Research Institute \\ and Hospital, National Cancer Center, Goyang, Seoul 10408, Republic of Korea
}

Received August 24, 2015; Accepted November 24, 2016

DOI: $10.3892 / 01.2017 .7308$

\begin{abstract}
Lymphatic endothelial cells (LEC) are major components of the tumor microenvironment and, due to the relative leakiness of lymphatic vessels compared with blood vessels, are essential for tumor dissemination and metastasis. In the present study, small interfering RNA-mediated suppression of E26 transformation-specific domain-containing protein Elk-3 (ELK3) inhibited the proliferation, migration and tube-forming ability of LEC. Suppression of ELK3 decreased vascular endothelial-cadherin expression levels and increased the phosphorylation of $\beta$-catenin. Furthermore, vascular endothelial growth factor receptor-3 (VEGFR-3) mRNA and protein expression levels were suppressed by the transfection of LEC with siELK3. As VEGFR-3 serves a major role in lymphangiogenesis, ELK3 may be a novel therapeutic target to inhibit tumor dissemination through the lymphatic system.
\end{abstract}

Correspondence to: Dr Kyung-Soon Park, Department of Biomedical Science, College of Life Science, CHA University, 335 Pangyo-ro, Bundang-gu, Seongnam-si, Seoul 13488, Republic of Korea

E-mail: kspark@cha.ac.kr

Dr Sun-Young Kong, Department of System Cancer Science, Graduate School of Cancer Science and Policy, National Cancer Center, 323 Ilsan-ro, Ilsandong-gu, Goyang-si, Seoul 10408, Republic of Korea

E-mail: ksy@ncc.re.kr

Abbreviations: ELK3, E26 transformation-specific domain-containing protein Elk-3; VEGFR-3, vascular endothelial growth factor receptor-3; LEC, lymphatic endothelial cells; LV, lymphatic vessels; ETS, E26 transformation-specific; HUVEC, human umbilical vein endothelial cells

Key words: E26 transformation-specific domain-containing protein Elk-3, lymphatic endothelial cell, vascular endothelial growth factor receptor-3, proliferation, migration

\section{Introduction}

E26 transformation-specific (ETS) domain-containing protein Elk-3 (ELK3) is a ternary complex factor belonging to the ETS transcription factor family. ELK3 was first identified as a transcriptional repressor of c-Fos, but it is able to function as a transcriptional activator once phosphorylated by the Ras/mitogen-activated protein kinase signaling pathway (1-3). The transcriptional activity of ELK3 has been linked to vasculogenesis and wound healing in cell lines and in mice (4-6). ELK3 regulates vascular integrity through early growth response protein 1 (4), and regulates angiogenesis through the control of vascular endothelial growth factor (VEGF) expression (6). Mice lacking ELK3 protein develop smaller tumors, which are not able to become vascularized and oxygenated, indicating that ELK3 serves a major role in tumorigenecity (6). In addition to phosphorylation, the transcriptional activity of ELK3 is regulated by nuclear-cytoplasmic shuttling in response to specific signaling pathways (7). ELK3 has two nuclear localization signals and it is primarily localized to the nucleus under physiological conditions (7). ELK3 also has a nuclear export signal, and the nuclear exclusion of ELK3 occurs via specific signaling pathways, including the c-Jun kinase pathway (7).

The tumor and organ microenvironment is important for cancer growth and metastasis. Diverse cells residing in the microenvironment influence cancer cells through secreted factors and their corresponding signaling pathways (8). These cell-to-cell communications contribute to cancer progression, angiogenesis, invasion, epithelial-to-mesenchymal transition phenotypes and antitumor drug resistance (9-11). Therefore, cells within the tumor microenvironment have emerged as attractive targets to effectively inhibit cancer. Lymphatic vessels (LV) are major components of the tumor microenvironment and are composed of lymphatic endothelial cells (LEC) (12). As LVs are more permeable than blood vessels, they are particularly important for tumor dissemination and metastasis (12). Despite the importance of LV in tumor progression, the factors secreted by LEC and the signals through which they influence the behavior of the tumor remain to be elucidated. 
The present study demonstrated that ELK3 regulates cell migration, tube formation and cell permeability of LEC. These results indicate that ELK3 is a major regulator of lymphangiogenesis in LEC.

\section{Materials and methods}

Cell culture. LEC and human umbilical vein endothelial cells (HUVEC) were purchased from Modern Cell \& Tissue Technologies (Seoul, Korea) and maintained in endothelial cell growth medium-2 supplemented with $5 \%$ fetal calf serum (FCS, \#CC-3202; Clonetics; Lonza, Basel, Switzerland) on surfaces coated with $2 \mu \mathrm{g} / \mathrm{ml}$ bovine fibronectin (Sigma-Aldrich; Merck KGaA, Darmstadt, Germany). The MDA-MB-231 and MCF7 breast cancer cell lines were cultured in Dulbecco's modified Eagle's medium supplemented with $10 \%$ fetal bovine serum (Thermo Fisher Scientific, Inc., Waltham, MA, USA).

Small interfering RNA (siRNA) transfection, RNA purification and reverse transcription-quantitative polymerase chain reaction $(R T-q P C R)$. The non-specific control siRNA (siNS; \#D-001810-10; Dharmacon, Inc., Chicago, IL, USA) and human ELK3 siRNA (siELK3; \#L-010320-00-0005; Dharmacon, Inc.) were obtained from Dharmacon (GE Healthcare Life Sciences, Chalfont, UK). The siRNAs (100 nM) were transfected into LEC using Lipofectamine ${ }^{\circledR} 2000$ (Thermo Fisher Scientific, Inc.) according to the manufacturer's protocol, and cells were collected at $48 \mathrm{~h}$ following transfection for further analysis. Total cellular RNA was extracted using TRIzol ${ }^{\circledR}$ reagent (Thermo Fisher Scientific, Inc., Invitrogen, Carlsbad, CA, USA) following the manufacturer's protocol. Total RNA (2 $\mu \mathrm{g})$ was used for single-stranded cDNA synthesis with OmniScript ${ }^{\circledR}$ reverse transcriptase (Qiagen $\mathrm{GmbH}$, Hilden, Germany). RT-qPCR was performed using the CFX96 Touch $^{\mathrm{TM}}$ Real-Time PCR Detection system (Bio-Rad Laboratories Inc., Hercules, CA, USA) using SYBR-Green I (Qiagen Inc., Valencia, CA, USA). The final volume of PCR mixture was $20 \mu \mathrm{l}$, comprising $1 \mu \mathrm{g}$ cDNA, 10 pmol forward and reverse primers and $10 \mu \mathrm{l}$ of $2 \mathrm{X}$ SYBR mix. The reactions were performed for 40 cycles using optimized cycling conditions (denaturation at $95^{\circ} \mathrm{C}$ for $1 \mathrm{~min}$, annealing at $55^{\circ} \mathrm{C}$ for $30 \mathrm{sec}$ and extension at $72^{\circ} \mathrm{C}$ for $30 \mathrm{sec}$ ). RT-qPCR data was processed based on the $2^{-\Delta \Delta \mathrm{Cq}}$ method (13). The primers used in this analysis are listed in Table I.

Protein extraction and western blotting. Total protein was isolated using Cell Lysis buffer (\#9803; Cell Signaling Technology Inc., Danvers, MA, USA) according to the manufacturer's protocol. A bovine serum albumin (BSA) standard curve was used to estimate the protein concentration. A total of 8 BSA standards were made by dilating $2 \mu \mathrm{g} / \mu \mathrm{l}$ albumin standard (\#23209; Thermo Fisher Scientific, Inc.) into cell lysis buffer, and they were then incubated at $37^{\circ} \mathrm{C}$ for $30 \mathrm{~min}$. A standard curve was produced based on the absorbance values of BSA solution in plate reader (Epoch microplate spectrophotometer; Biotek Instruments, Inc., Winooski, VT, USA) at $562 \mathrm{~nm}$. Total protein $(20 \mu \mathrm{g})$ was separated by $10 \%$ SDS-PAGE and transferred to a polyvinylidene difluoride (PVDF) membrane (EMD Millipore, Billerica, MA, USA). The membrane was blocked by $5 \%$ skim milk solution at
Table I. Reverse transcription-quantitative polymerase chain reaction primers.

\begin{tabular}{ll} 
Gene & \multicolumn{1}{c}{ Primer sequence (5'-3') } \\
\hline GAPDH & F-ACCACAGTCCATGCCATCAC \\
& R-TCCACCACCCTGTTGCTGTA \\
ELK3 & F-ACCCAAAGGCTTGGAAATCT \\
& R-TGTATGCTGGAGGACAGTGG \\
VE- & F-TCCTCTGCATCCTCACTATCACA \\
cadherin & R-GTAAGTGACCAACTGCTCGTGAAT \\
LYVE1 & F-ACTTCCATCTGGACCACGAG \\
& R-AGCCTACAGGCCTCCTTAGC \\
PROX1 & F-CAGCCCGAAAAGAACAGAAG \\
& R-GGGTCTAGCTCGCACATCTC \\
$V E G F R 1$ & F-GCTGTGCGCGCTGCTT \\
& R-AACTCAGTTCAGGACCTTTAATTTTGA \\
$V E G F R 2$ & F-GTGACCAACATGGAGTCGTG \\
& R-TGCTTCACAGAAGACCATGC \\
$V E G F R 3$ & F-GAGACAAGGACAGCGAGGAC \\
& R-TCACGAACACGTAGGAGCTG \\
$V E G F A$ & F-GAGGATCAAACCTCACCAAG \\
& R-CCGCCTCGGCTTGTCACAT \\
$V E G F B$ & F-CCCTTGACTGTGGAGCTCAT \\
$V E G F C ~$ & R-GGCTTCACAGCACTGTCCTT \\
& F-ACCAAACAAGGAGCTGGATG \\
& R-ATTTCTGGGGCAGGTTCTTT \\
& F-AGGACTGGAAGCTGTGGAGA \\
& R-ATCGGAACACGTTCACACAA \\
&
\end{tabular}

F, forward; R, reverse; VEGF, vascular endothelial growth factor; VE-cadherin, vascular endothelial-cadherin, PROX1, prospero homeobox 1; VEGFR, vascular endothelial growth factor receptor; LYVE1, lymphatic vessel endothelial hyaluronan receptor 1; GAPDH, glyceraldehyde-3-phosphate dehydrogenase; ELK3, ETS transcription factor 3 .

room temperature for $30 \mathrm{~min}$ and then incubated with an anti-ELK3 antibody (\#sc-17860, 1:1,000 dilution; Santa Cruz Biotechnology Inc., Dallas, TX, USA), an antibody recognizing active $\beta$-catenin (unphosphorylated at Ser33/37/Thr41, \#4270S), an anti-phospho- $\beta$-catenin antibody (phosphorylated at Ser33/37/Thr41, \#9561S) (both from Cell Signaling Technology, Inc.), an anti-lamin B antibody (\#sc-6216) or an anti- $\beta$-actin antibody (\#sc-47778) (both from Santa Cruz Biotechnology, Inc.) overnight at $4^{\circ} \mathrm{C}$, followed by incubation with a secondary antibody (\#sc-2005, 1:2,000 dilution; Santa Cruz Biotechnology, Inc.) for $1 \mathrm{~h}$ at room temperature. The membrane was washed three times in TBST for $5 \mathrm{~min}$ each time. Immunoreactive proteins were detected using the SuperSignal West Pico Chemiluminescent Substrate Western Blot Detection system (catalog no. 34080; Thermo Fisher Scientific, Inc.) according to the protocol of the manufacturer. All experiments were repeated at least three times.

$2 D$ migration assay. The migration potential of LEC was assessed using a wound healing assay. LEC, following $24 \mathrm{~h}$ transfection with siNS or siELK3, were cultured on $26 \times 76 \mathrm{~mm}$ 
A

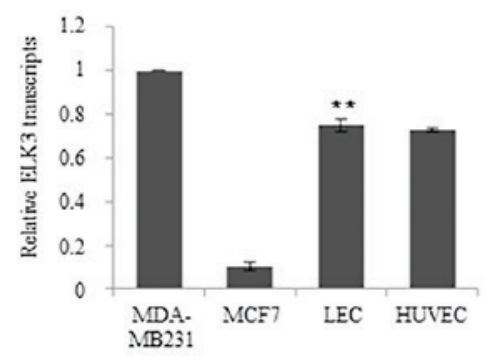

C

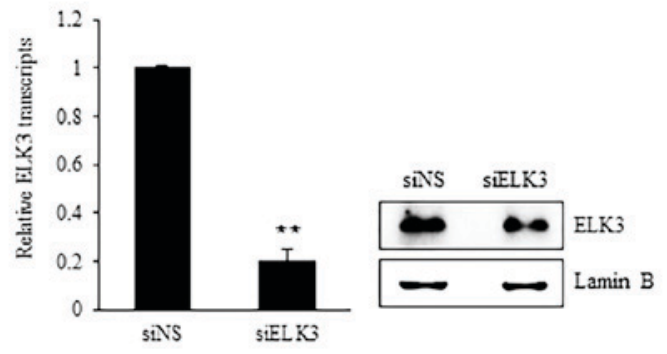

B

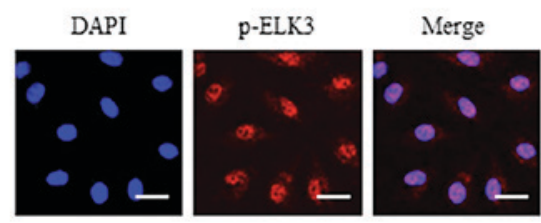

D

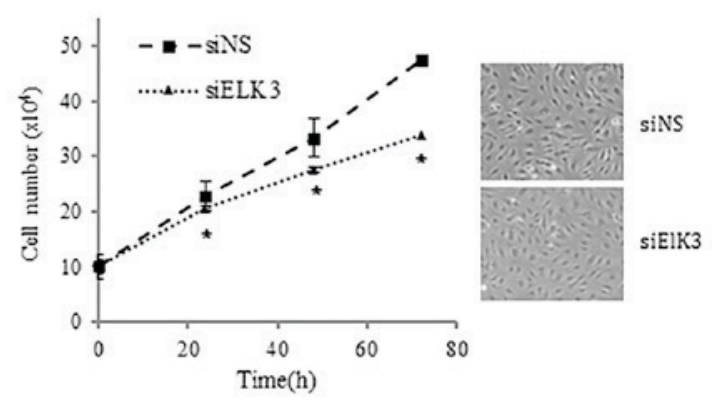

Figure 1. The role of ELK3 in the proliferation of LEC. (A) ELK3 expression evaluated using reverse transcription-quantitative polymerase chain reaction in LEC, HUVEC, MCF7 and MDA-MB-231 breast cancer cell line. (B) Immunocytochemical staining for ELK3 in LEC using DAPI and an anti-p-ELK3 antibody (scale bar, $20 \mathrm{um}$ ). (C) Suppression of ELK3 expression by transfection with siELK3 or siNS into LEC. (D) Morphology of LEC transfected with siELK3 or siNS was observed using light microscopy and cell proliferation was analyzed using an MTT assay at the indicated time points. All values are the mean \pm standard deviation from $\geq 3$ independent experiments. ${ }^{*} \mathrm{P}<0.05$, Student's t-test. ELK3, E26 transformation-specific domain-containing protein Elk-3; siNS, non-specific siRNA; siELK3, siRNA-targeting ELK3; HUVEC, human umbilical vein endothelial cells; LEC, lymphatic endothelial cells.

glass coverslips in endothelial cell growth medium-2 supplemented with $5 \%$ FCS and wounded using a micropipette tip when the cells were fully confluent. The cells were incubated for $24 \mathrm{~h}$ at $37^{\circ} \mathrm{C}$ and then observed for migration using a light microscope (CKX41SF; Olympus, Tokyo, Japan).

In vitro tube formation assay. The ability of LEC to form capillary-like structures (tubes) was assessed using a tube formation assay (14). LEC were harvested and cultured in media with or without recombinant VEGF-C for $30 \mathrm{~min}$ at room temperature. A total of $1.5 \times 10^{4}$ LEC cells were seeded on slides coated with Matrigel (\#356231; Corning Inc., Corning, NY, USA) supplemented with vitronectin (SPR3186; Sigma-Aldrich; Merck KGaA) (5 mg/ml). Cells were incubated for $24 \mathrm{~h}$ in the presence of $5 \% \mathrm{CO}_{2}$ at $37^{\circ} \mathrm{C}$ for further analysis.

Cell proliferation assay. Cell proliferation was analyzed using the MTT assay. Briefly, 3x10 3 cells were seeded onto 96 -well plates and cultured for 24,48 and $72 \mathrm{~h}$ at $37^{\circ} \mathrm{C}$. MTT solution was added to each well at a final concentration of $0.5 \mathrm{mg} / \mathrm{ml}$ and incubated for $4 \mathrm{~h}$ at $37^{\circ} \mathrm{C}$. The resulting formazan crystals were dissolved in $150 \mu \mathrm{l}$ dimethyl sulfoxide per well. The absorbance was measured at a wavelength of $570 \mathrm{~nm}$ using an automated plate reader (Thermo Fisher Scientific, Inc.).

Immunocytochemical staining. Immunocytochemical staining was performed as described previously (15), using a rabbit anti-human VE-cadherin antibody (\#sc-9989; dilution, 1:200; Santa Cruz Biotechnology, Inc.) and an Alexa Fluor ${ }^{\circledR}$ 488-conjugated secondary antibody (\#A21206; 1:300 dilution; Thermo Fisher Scientific, Inc.) at room temperature. Nuclei were stained by incubating cells with $5 \mu \mathrm{g} / \mathrm{ml}$ DAPI labeling solution (\#ab104140; Abcam, Cambridge, UK) for 2-5 min in the dark at room temperature.

Analysis of VEGF-C protein in the culture media. The amount of $V E G F-C$ in the culture media was measured by VEGF-C Quantikine ELISA kit (R\&D Systems, Inc., Minneapolis, USA; \#DVEC00) according to the protocol of the manufacturer.

Statistical analysis. Graphical data are presented as the means \pm standard deviation. $\mathrm{P}<0.05$ and $\mathrm{P}<0.01$ were considered to indicate significant and highly significant results based on Student's t-test analyses, respectively. Statistical analyses were performed using the SAS statistical package v.9.13 (SAS Institute, Cary, NC, USA; http:// www.sas.com/).

\section{Results}

ELK3 regulates the proliferation of LEC. ELK3 is highly expressed in the LV of mice (13). The expression of ELK3 in LEC was examined to elucidate the role of ELK3 in lymphangiogenesis. The expression levels of ELK3 in LEC were comparable to those in HUVEC cells and in the MDA-MB-231 and MCF-7 breast cancer cell line, in which ELK3 has been established to be important in angiogenesis (Fig. 1A). ELK3 is 

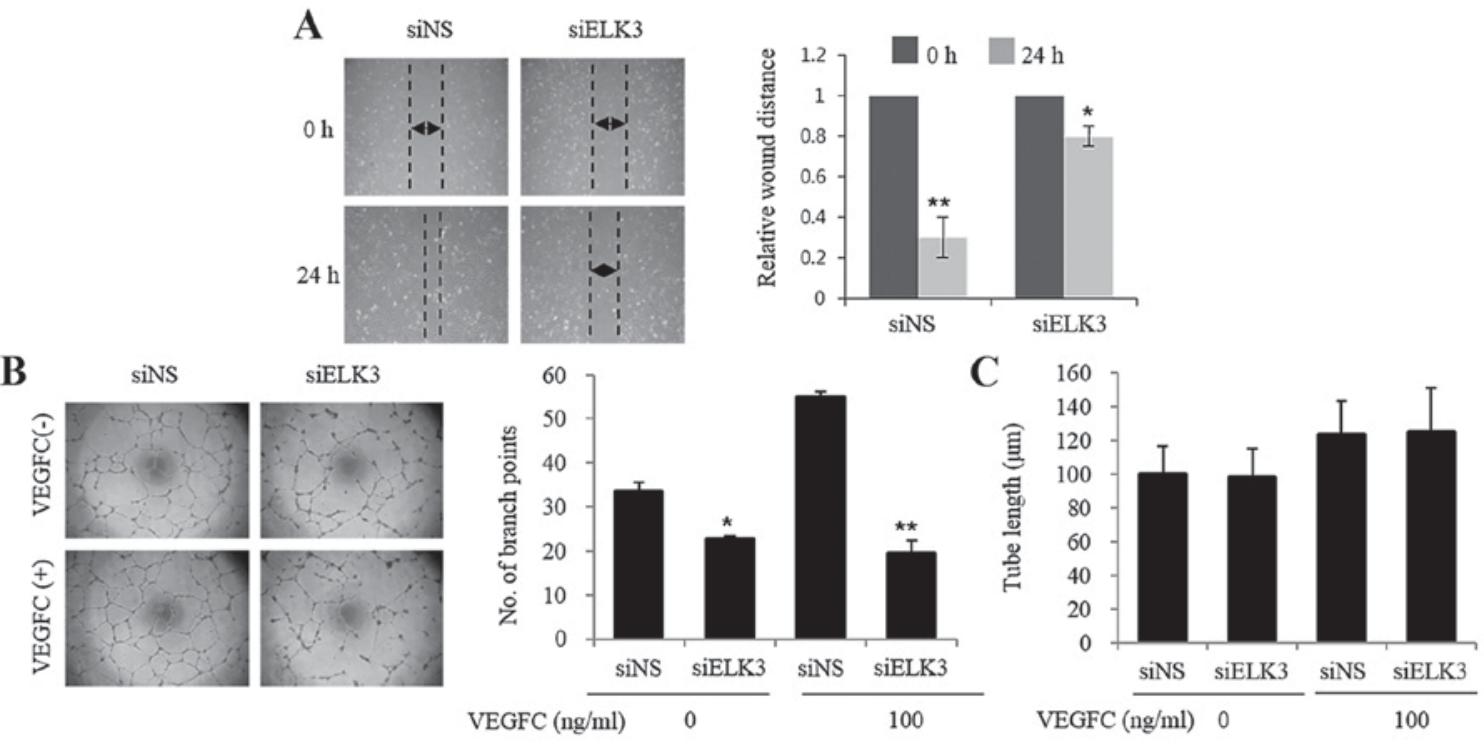

Figure 2. ELK3 regulates the migration and tube-forming abilities of LEC. (A) LEC transfected with siELK3 or siNS for $24 \mathrm{~h}$ were analyzed for their migration potential by a scratch assay. Migration was observed $24 \mathrm{~h}$ following wounding. (B) Tube formation by LEC transfected with siELK3 or siNS was observed using microscopy. The number of branch points is quantified in the graph. (C) The relative tube length was measured and depicted in the graph. All values are the mean \pm standard deviation from $\geq 3$ independent experiments. " $\mathrm{P}<0.05,{ }^{* * *} \mathrm{P}<0.01$, Student's t-test. ELK3, E26 transformation-specific domain-containing protein Elk-3; siNS, non-specific siRNA; siELK3, siRNA-targeting ELK3; LEC, lymphatic endothelial cells; VEGFC, vascular endothelial growth factor C.

primarily localized to the nucleus unless it is actively exported to the cytoplasm via specific signaling molecules, including c-Jun (16). As presented in Fig. 1B, the phosphorylated form of ELK3 was detected in the nucleus, indicating that ELK3 may function as an active transcription factor in LEC. To examine the role of ELK3 in LEC, the protein expression of ELK3 was suppressed using siRNA (Fig. 1C). Suppression of ELK3 protein expression did not exhibit any effect on LEC morphology but did result in a decreased proliferation rate ( $\mathrm{P}<0.05$; Fig. 1D).

ELK3 regulates migration and tube formation of LEC. An in vitro scratch assay that mimics cell migration into an artificial wound produced on a cell monolayer was used to investigate the role of ELK3 in LEC migration (17). The healing ability of LEC was inhibited at $24 \mathrm{~h}$ following wounding in cells transfected with siELK3 (Fig. 2A). The effect of ELK3 suppression on the vascular behavior of LEC was evaluated using a tube formation assay. As presented in Fig. 2B, LEC transfected with siELK3 formed fewer branch points compared with those in the siNS controls in the presence and absence of VEGF-C $\left({ }^{*} \mathrm{P}<0.05,{ }^{* *} \mathrm{P}<0.01\right)$. However, tube length was not affected by the silencing of ELK3 (Fig. 2C). These results suggest that ELK3 may regulate the vasculogenic activity of LEC.

Expression of VE-cadherin and VEGFR-3 and the phosphorylation of $\beta$-catenin, are regulated by ELK3. Endothelial permeability is closely associated with the dissemination of cancer cells and is, therefore, particularly important in cancer biology (18). As the expression of VE-cadherin regulates endothelial permeability and mediates cell-to-cell contact (19), the expression levels of VE-cadherin were analyzed in the presence and absence of siELK3. As presented in Fig. 3A, the expression of VE-cadherin mRNA was decreased following transfection with siELK3 $(\mathrm{P}<0.05)$. The accumulation of VE-cadherin near the membrane and at points of cell-to-cell contact was lower in siELK3-transfected LEC compared with siNS control cells $(\mathrm{P}<0.05$, Fig. $3 \mathrm{~B})$. These results suggest that ELK3 may function as a positive regulator of VE-cadherin expression levels in LEC. In addition to the expression of VE-cadherin, the phosphorylation of $\beta$-catenin has also been implicated in VE-cadherin-mediated cell adhesion (20-22). As the phosphorylation of $\beta$-catenin correlates with the loss of VE-cadherin function (23), the phosphorylation of $\beta$-catenin was compared in siELK3-transfected and control LEC in the presence and absence of VEGF-C. Notably, ELK3 suppression was correlated with the phosphorylation of $\beta$-catenin in the presence and absence of VEGF-C (Fig. 3C). These results suggest that ELK3 may regulate the transcriptional expression of VE-cadherin as well as the phosphorylation of $\beta$-catenin.

In the tumor microenvironment, particularly in breast cancer, LV is a predominant route of tumor dissemination (24). Tumor lymphangiogenesis is driven by growth factors, including VEGF-C and VEGF-D, which are secreted from tumor cells (25). LEC in the LV also secrete factors into the tumor microenvironment that facilitate tumor dissemination (24). Therefore, the effect of ELK3 suppression on the expression levels of angiogenic factors was analyzed by RT-qPCR. VEGFR-3, which serves an important role in lymphangiogenesis as the receptor for VEGF-C, was significantly downregulated, whereas the expression of VECF-C was upregulated in siELK3-transfected LEC (Fig. 3D). Consistent with the mRNA levels, the quantity of VEGF-C protein in the culture media of siELK3-transfected LEC was higher compared with the controls $(\mathrm{P}<0.05$, Fig. 3E). These results demonstrate that ELK3 suppression may make LEC insensitive to VEGF-C stimulation, possibly via the reduced expression of VEGFR-3. 
A

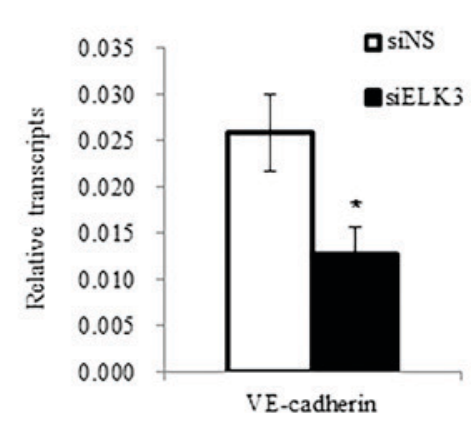

D

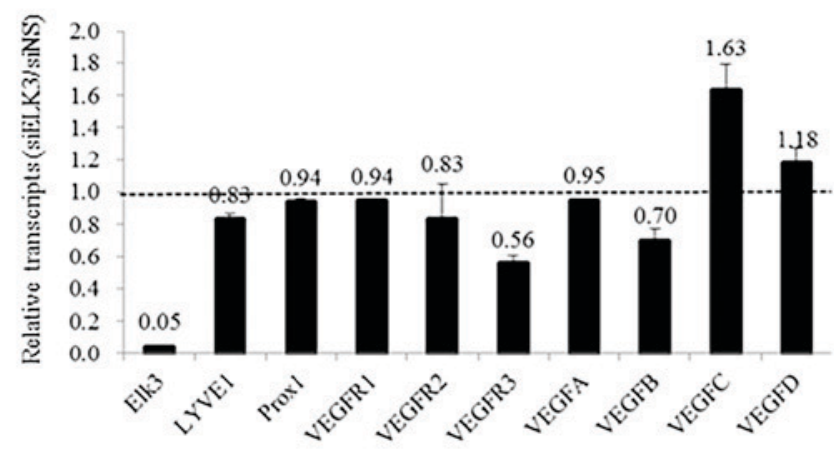

B

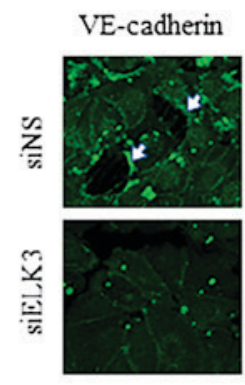

C

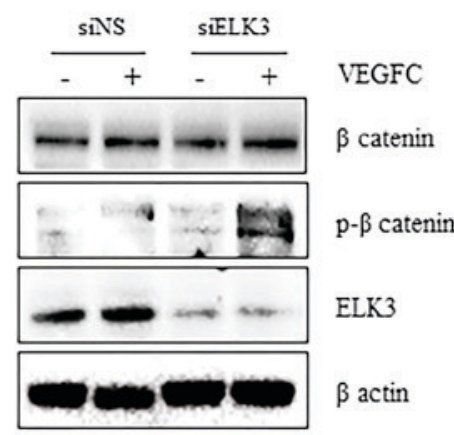

$\mathbf{E}$
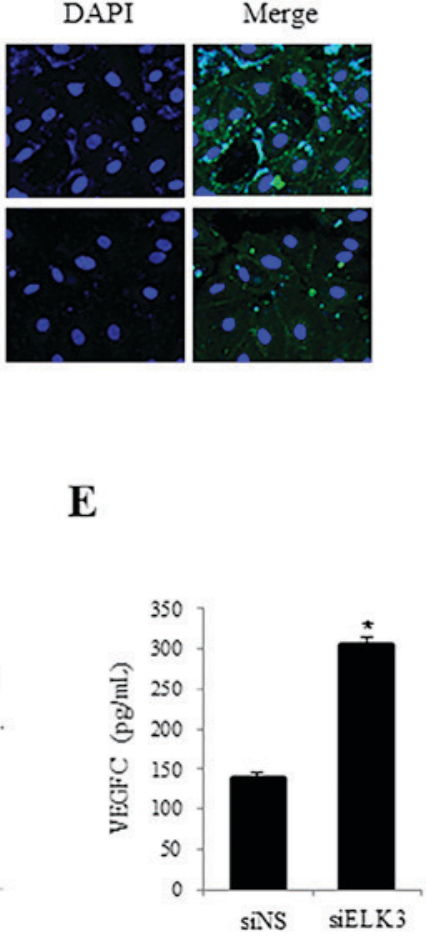

Figure 3. ELK3 regulates the expression of VE-cadherin and VEGFR-3, and the phosphorylation of $\beta$-catenin. (A) VE-cadherin expression in LEC transfected with siELK3 and siNS for $48 \mathrm{~h}$ was evaluated using RT-qPCR. (B) VE-cadherin expression was detected by immunocytochemical staining in LEC transfected with siELK3 or siNS for $24 \mathrm{~h}$. Arrows indicates VE-cadherin staining. (C) Accumulation of phosphorylated $\beta$-catenin was analyzed using western blot analysis. LEC were transfected with siNS or siELK3 for $24 \mathrm{~h}$ and untreated or stimulated with VEGF-C for 30 min. (D) The effect of ELK3 knockdown on the expression of the indicated genes was evaluated using real-time RT-qPCR. LEC were transfected with siELK3 for $24 \mathrm{~h}$ and the expression of each gene is presented relative to the levels in control (siNS) cells (siELK3/siNS). (E) The quantity of VEGF-C was determined using ELISA. ELK3, E26 transformation-specific domain-containing protein Elk-3; siNS, small interfering RNA non-specific control; siELK3, small interfering RNA ELK3; VE-cadherin, vascular endothelial-cadherin; LEC, lymphatic endothelial cells; VEGF-C, vascular endothelial growth factor C; RT-qPCR, reverse transcription quantitative polymerase chain reaction.

\section{Discussion}

The present study demonstrated that ELK3 may be involved in the proliferation, migration and tube formation by LEC, activities that are required for lymphangiogenesis (14). In particular, the results suggested that ELK3 positively regulates VEGFR-3 expression in LEC. Other ETS family transcription factors possess the ability to induce lymphangiogenesis by regulating VEGFR-3 (26). However, the current study, to the best of our knowledge, is the first report that ELK3 may regulate VEGFR-3 expression levels.

The lymphatic endothelium functions as a selective permeable barrier controlling transfer between vessel and tissues (27). Impaired endothelium permeability results in persistent vascular leakage, and is implicated in diverse pathological conditions (28). Mice that express a mutant version of ELK3 lacking the ETS DNA-binding domain develop dilated LV (4). This is concordant with the results of the current study, which suggest that ELK3 may be implicated in LEC permeability. It is also important to evaluate whether VEGFR-3 downstream signaling is regulated by ELK3, as the binding of ligands including VEGF-C to VEGFR-3 activates the phosphoinositide 3-kinase/protein kinase $\mathrm{B}$
(PI3-K/Akt) signaling pathway, and the physical interaction between VEGFR-3 and PI3 $\mathrm{K}$ is associated with lymph node metastasis (29).

In conclusion, it is therefore important for future studies to elucidate whether alterations in ELK3 expression are associated with disturbances in PI3-K/Akt signaling. As the VEGFR-3/PI3-K signaling pathway is implicated in lymphangiogenesis in the LEC, the ELK3-VEFGR3-PI3K axis may be a novel therapeutic target to inhibit tumor dissemination through the lymphatic system.

\section{Acknowledgements}

The present study was supported by the Korea Science and Engineering Foundation of the Korean government (grant no. 2015R1A2A2A01003498). Ministry of Education, Science, and Technology (NRF-2017-M3A9B4031169).

\section{References}

1. Criqui-Filipe P, Ducret C, Maira SM and Wasylyk B: Net, a negative Ras-switchable TCF, contains a second inhibition domain, the CID, that mediates repression through interactions with CtBP and de-acetylation. EMBO J 18: 3392-3403, 1999. 
2. Giovane A, Pintzas A, Maira SM, Sobieszczuk P and Wasylyk B Net, a new ets transcription factor that is activated by Ras. Genes Dev 8: 1502-1513, 1994.

3. Maira SM, Wurtz JM and Wasylyk B: Net (ERP/SAP2) one of the Ras-inducible TCFs, has a novel inhibitory domain with resemblance to the helix-loop-helix motif. EMBO J 15: 5849-5865, 1996

4. Ayadi A, Zheng H, Sobieszczuk P, Buchwalter G, Moerman P, Alitalo $\mathrm{K}$ and Wasylyk B: Net-targeted mutant mice develop a vascular phenotype and up-regulate egr-1. EMBO J 20: 5139-5152, 2001.

5. Nozaki M, Onishi Y, Kanno N, Ono Y and Fujimura Y: Molecular cloning of Elk-3, a new member of the Ets family expressed during mouse embryogenesis and analysis of its transcriptional repression activity. DNA Cell Biol 15: 855-862, 1996.

6. Zheng H, Wasylyk C, Ayadi A, Abecassis J, Schalken JA, Rogatsch H, Wernert N, Maira SM, Multon MC and Wasylyk B: The transcription factor Net regulates the angiogenic switch. Genes Dev 17: 2283-2297, 2003.

7. Ducret C, Maira SM,Dierich A and Wasylyk B: The net repressor is regulated by nuclear export in response to anisomycin, UV, and heat shock. Mol Cell Biol 19: 7076-7087, 1999.

8. Chen ST, Pan TL, Juan HF, Chen TY, Lin YS and Huang CM Breast tumor microenvironment: Proteomics highlights the treatments targeting secretome. J Proteome Res 7: 1379-1387, 2008.

9. Whiteside TL: The tumor microenvironment and its role in promoting tumor growth. Oncogene 27: 5904-5912, 2008.

10. Funasaka T and Raz A: The role of autocrine motility factor in tumor and tumor microenvironment. Cancer Metastasis Rev 26 : 725-735, 2007.

11. Tredan O, Galmarini CM, Patel K and Tannock IF: Drug resistance and the solid tumor microenvironment. J Natl Cancer Inst 99: 1441-1454, 2007.

12. Yong LC and Jones BE: A comparative study of cultured vascular and lymphatic endothelium. Exp Pathol 42: 11-25, 1991.

13. Livak KJ and Schmittgen TD: Analysis of relative gene expression data using real-time quantitative PCR and the 2(-Delta Delta C(T)) method. Methods 25: 402-408, 2001

14. Heo SH and Cho JY: ELK3 suppresses angiogenesis by inhibiting the transcriptional activity of ETS-1 on MT1-MMP. Int J Biol Sci 10: 438-447, 2014

15. Park KS, Cha Y, Kim CH, Ahn HJ, Kim D, Ko S, Kim KH, Chang MY, Ko JH, Noh YS, et al: Transcription elongation factor Tcea3 regulates the pluripotent differentiation potential of mouse embryonic stem cells via the Lefty1-Nodal-Smad2 pathway. Stem Cells 31: 282-292, 2013.

16. Johnson GL and Nakamura K: The c-jun kinase/stress-activated pathway: Regulation, function, and role in human disease. Biochim Biophys Acta 1773: 1341-1348, 2007.

17. Liang CC, Park AY and Guan JL: In vitro scratch assay: A convenient and inexpensive method for analysis of cell migration in vitro. Nat Protoc 2: 329-333, 2007.
18. Nishida N, Yano H, Nishida T, Kamura T and Kojiro M: Angiogenesis in cancer. Vasc Health Risk Manag 2: 213-219, 2006.

19. Gavard J: Endothelial permeability and VE-cadherin: A wacky comradeship. Cell Adh Migr 8: 158-164, 2014.

20. Lilien J and Balsamo J: The regulation of cadherin-mediated adhesion by tyrosine phosphorylation/dephosphorylation of beta-catenin. Curr Opin Cell Biol 17: 459-465, 2005.

21. Monaghan-Benson E and Burridge K: The regulation of vascular endothelial growth factor-induced microvascular permeability requires Rac and reactive oxygen species. J Biol Chem 284: 25602-25611, 2009.

22. Winter MC, Shasby S and Shasby DM: Compromised E-cadherin adhesion and epithelial barrier function with activation of $\mathrm{G}$ protein-coupled receptors is rescued by Y-to-F mutations in beta-catenin. Am J Physiol Lung Cell Mol Physiol 294: L442-L448, 2008.

23. Timmerman I, Hoogenboezem M, Bennett AM, Geerts D, Hordijk PL and van Buul JD: The tyrosine phosphatase SHP2 regulates recovery of endothelial adherens junctions through control of $\beta$-catenin phosphorylation. Mol Biol Cell 23: 4212-4225, 2012.

24. Shayan R, Achen MG and Stacker SA: Lymphatic vessels in cancer metastasis: Bridging the gaps. Carcinogenesis 27: 1729-1738, 2006.

25. Cao Y: Opinion: Emerging mechanisms of tumour lymphangiogenesis and lymphatic metastasis. Nat Rev Cancer 5: 735-743, 2005.

26. Yoshimatsu Y, Yamazaki T, Mihira H, Itoh T, Suehiro J, Yuki K, Harada K, Morikawa M, Iwata C, Minami T, et al: Ets family members induce lymphangiogenesis through physical and functional interaction with Prox1. J Cell Sci 124: 2753-2762, 2011.

27. Sukriti S, Tauseef M, Yazbeck P and Mehta D: Mechanisms regulating endothelial permeability. Pulm Circ 4: 535-551, 2014.

28. Arima M, Cui D, Kimura T, Sonoda KH, Ishibashi T, Matsuda S and Ikeda E: Basigin can be a therapeutic target to restore the retinal vascular barrier function in the mouse model of diabetic retinopathy. Sci Rep 6: 38445, 2016

29. Coso S, Zeng Y, Opeskin K and Williams ED: Vascular endothelial growth factor receptor-3 directly interacts with phosphatidylinositol 3-kinase to regulate lymphangiogenesis. PLoS One 7: e39558, 2012.

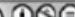

This work is licensed under a Creative Commons Attribution-NonCommercial-NoDerivatives 4.0 International (CC BY-NC-ND 4.0) License. 